ORIGINAL

\title{
Diagnóstico clínico-microbiológico de otitis externa en caninos de Bogotá - Colombia
}

\section{Clinical - microbiological diagnostic of external otitis in canines in Bogotá - Colombia}

\author{
Adriana Pulido V,${ }^{1}$ M.Sc, Rubiela Castañeda $S,{ }^{1}$ M.Sc, Melva Linares $L,{ }^{2}$ M.Sc(c), Marcela \\ Mercado G, ${ }^{3}$ M.Sc. \\ ${ }^{1}$ Pontificia Universidad Javeriana (PUJ). Facultad de Ciencias (FC). Departamento de Microbiología (Mic). \\ Área de diagnóstico veterinario. ${ }^{2} \mathrm{PUJ}-\mathrm{FC}-\mathrm{Mic}$. Área de Micología. ${ }^{3} \mathrm{PUJ}-\mathrm{FC}-\mathrm{Mic}$. Área de Epidemiología. \\ Bogotá D.C. Colombia. *Correspondencia: adriana.pulido@javeriana.edu.co
}

Recibido: Septiembre 2 de 2009; Aceptado: Marzo 21 de 2010.

\section{RESUMEN}

Objetivo. Evaluar clínica y microbiológicamente la implicación de Malassezia sp como agente etiológico de otitis externas en caninos. Materiales y métodos. Se obtuvieron 166 muestras de hisopados óticos de caninos con sintomatología y hallazgos clínicos compatibles con otitis externa durante el periodo comprendido entre julio - diciembre 2008. A partir de las muestras se realizaron cultivos bacteriológicos y micológicos e identificación de género mediante perfiles bioquímicos para cada unos de los microorganismos aislados. Los datos clínicos asociados a la patología fueron consignados en una base de datos y posteriormente analizados en el programa estadístico SPSS 17 Resultados. A partir de la 166 muestras obtenidas, 59\% (98) de ellas fueron positivas para el cultivo bacteriológico con predominio de Staphylococcus sp 37\% (61) y 73\% (121) positivas para Malassezia. De las 121 levaduras aisladas 32.2\% fue Malassezia pachydermatis. El análisis estadístico no evidenció diferencias significativas con respecto a las relaciones entre variables (género, edad, raza, tipo de oreja y respuesta inflamatoria por citología) y el aislamiento de Malassezia sp. El nivel de significancia establecido para la prueba fue de $0.05 \%$. Conclusiones. No se observaron asociaciones estadísticas entre las variables género, edad, raza frente a la otitis externa por Malassezia sp. Malassezia sp fue aislada en un $73 \%$ de los casos con otitis externa, con participación de diferentes agentes bacterianos especialmente Staphylococcus sp.

Palabras clave: Canino, otitis externa, Malassezia sp., Staphylococcus sp. 


\section{ABSTRACT}

Objective. To evaluate clinically and microbiologically implication of Malassezia sp as an etiologic agent of external otitis in canines. Materials and methods. 166 samples of otic swabs in canines with symptoms and clinical findings consistent with external otitis were obtained during the period between July and December 2008. Bacteriological and mycological cultures were carried out from the samples, identification using biochemical profiles for each of the isolated microorganisms were done. Clinical data associated with the disease was entered into a database and then analyzed with the SPSS 17 statistical program. Results. From the 166 samples 98, (59\%) of them were positive for bacterial culture with a predominance of Staphylococcus sp 37\% (61) and 121 (73\%) were positive for Malassezia. Of the 121 yeast isolates, 32.2\% were Malassezia pachydermatis. Statistical analysis showed no significant difference regarding the relationship between variables (gender, age, race, type of ear or inflammatory response by cytology) and the isolation of Malassezia sp. The significance level for the test was set on $0.05 \%$. Conclusions. There were no statistical associations between the gender, age, or race variables and external otitis by Malassezia sp. Malassezia sp was isolated in $73 \%$ of patients with external otitis, involving different bacterial agents especially Staphylococcus sp.

Key words: Canine, external otitis, Malassezia sp., Staphylococcus sp.

\section{INTRODUCCIÓN}

Las enfermedades óticas son entidades de frecuente presentación en medicina de pequeños animales, siendo la otitis externa una de las patologías auditivas más comúnmente diagnosticadas, ésta se define como una inflamación del canal auditivo externo (1-3).

Dentro de las posibles etiologías de otitis externa se encuentran en primer lugar causas primarias de la enfermedad como son cuerpos extraños y presencia de parásitos dentro del canal, enfermedades de tipo alérgico y/o autoinmune y desórdenes de la queratinización principalmente; en segundo lugar existen factores predisponentes como el tamaño y la posición de la oreja, humedad excesiva de la misma, factores iatrogénicos, y disminución de la luz del canal auditivo lo que impide un adecuado drenaje de las secreciones óticas $(2,3)$. Adicionalmente existen otros factores asociados a la enfermedad como son la presencia de bacterias y/o levaduras así como los procesos infecciosos del oído medio que juegan un papel importante en los casos de otitis recurrentes (2).

Uno de los principales agentes etiológicos involucrados con los procesos de otitis externa en caninos son las levaduras, dentro de las cuales se encuentran implicadas aquellas pertenecientes al género Malassezia. Malassezia es una levadura lipofílica considerada como flora cutánea y ótica normal, la cual puede convertirse en patógena de acuerdo con las condiciones microambientales del canal auditivo del hospedero y a las alteraciones del sistema inmunológico $(2,4-7)$.

A excepción de M. pachydermatis todas las levaduras pertenecientes al género Malassezia son lipodependientes (4, 8-10) ya que utilizan ácidos grasos de cadena larga (ácido oléico, linoléico, palmítico, mirístico) como fuente de carbono lo que hace necesaria su inclusión en los medios de cultivo artificiales como el Dixon $(5,6,10,11)$ y Leming's que garantizan un óptimo crecimiento $(12,13)$. La temperatura ideal para su desarrollo es de $32^{\circ} \mathrm{C}\left(31-35^{\circ} \mathrm{C}\right)$ por un periodo de incubación de 7 días, después de los cuales se observan características macroscópicas de las colonias como: color blanco-amarillento, pequeñas, lisas o levemente rugosas, brillantes o mates; sin embargo, microscópicamente se observan algunas variaciones en forma y tamaño entre especies; junto con las pruebas de 
catalasa, ureasa, esculina y patrones de asimilación de lípidos es posible realizar la identificación de especie $(8,10,12,14)$.

Otros patógenos importantes en los procesos óticos son las bacterias tanto Gram positivas como Gram negativas, de las cuales algunas se pueden encontrar como flora normal del canal auditivo pero que bajo condiciones que permitan una colonización masiva ocasionan un proceso patológico; las más comúnmente asociadas con otitis son Staphylococcus intermedius, Pseudomonas aeruginosa (importante en otitis crónica), Proteus mirabilis, Escherichia coli, Corynebacterium spp, Enterococcus spp, y Streptococcus spp $(2,8)$.

En estudios preliminares realizados por el grupo de investigación UNIDIA de la Pontificia Universidad Javeriana, se detectó en la ciudad de Bogotá una prevalencia de consultas por problemas óticos del $20 \%$, del cual el $40 \%$ estuvo relacionado con otitis externa $y / u$ otitis causadas por levaduras; llegando al diagnóstico definitivo solo por el examen clínico (75\%) y en ocasiones con citología exfoliativa (15\%). Teniendo en cuenta este análisis, el objetivo del trabajo fue realizar el diagnóstico microbiológico en pacientes que fueron llevados a consulta en cuatro (4) clínicas veterinarias de Bogotá, durante el periodo de julio - diciembre de 2008, y de esta manera realizar el primer reporte en el país sobre aislamientos de levaduras del género Malassezia spp asociadas a patologías óticas en caninos.

\section{MATERIALES Y MÉTODOS}

Recolección de datos. Para la obtención de los anamnésicos de los pacientes se diseñó un formulario en el que se consignaron los datos correspondientes a edad, género, raza, antecedentes de problemas óticos, realización de tratamientos caseros y/o farmacológicos para ser analizados y determinar su correlación con la presentación del cuadro clínico.

Obtención de las muestras. Se seleccionaron 4 clínicas veterinarias en la ciudad de Bogotá, en las cuales se hicieron los muestreos por un periodo de seis meses. Se obtuvieron 166 hisopados óticos de caninos que presentaban sintomatología compatible con otitis externa. La muestra de la(s) oreja(s) afectadas se obtuvo con un hisopo de algodón estéril (cultturete $\AA$ ) previamente impregnado con solución de transporte Tween 20 al 0.05\%, garantizando así un ambiente lipofílico, el cual fue utilizado para cultivo. Se tomó un segundo hisopo para análisis citológico y evaluación bacteriológica.

Cepas de referencia. Se utilizaron las cepas CBS: Malassezia furfur 7019, Malassezia pachydermatis 1879, Malassezia symphodialis 7222, Malassezia restricta 7877, Malassezia slooffiae 7956, Malassezia globosa 7966.

Aislamiento bacteriano. Las muestras se sembraron en agar sangre para la evaluación y clasificación del crecimiento bacteriano.

\section{Procesamiento de las muestras.}

\section{Aislamiento primario e identificación} bioquímica. Las muestras se sembraron en agar sangre, se incubaron a $37^{\circ} \mathrm{C}$ por 24-48 horas y se procedió a la identificación bacteriológica siguiendo los estándares de clasificación; adicionalmente para el aislamiento de levaduras se utilizó agar Sabouraud y agar Dixon's, fueron incubados a $32^{\circ} \mathrm{C}$ evaluándose crecimiento a los 2, 5 y 7 días, una vez identificadas las características morfológicas de crecimiento, se realizaron pruebas bioquímicas como determinación de ureasa, hidrólisis de bilis esculina $(10,15)$, crecimiento en agar Sabouraud, asimilación de Tween 20,40,60 y $80(10,11)$ y asimilación del cremophor (16).

Citología exfoliativa. Se realizó coloración de Gram y Wright para determinar la presencia o ausencia de bacterias, levaduras y reacción inflamatoria. La lectura se realizó teniendo en cuenta la escala semicuantitativa por cruces (de $+\mathrm{a}++++$ ) indicando el tipo de morfología bacteriana y su ordenamiento; para levaduras se tuvo en cuenta la escala propuesta por Nobre et al (17) con algunas modificaciones de acuerdo a lo observado en el medio de estudio, 
negativo, + de 1 a 5 células/campo, ++ de 6 - 10 células/campo, +++ de 10 a 19 células por campo y $++++>19$ células/ campo (17).

Análisis estadístico. Se realizó un estudio prospectivo, descriptivo y transversal. Los datos fueron analizados utilizando el programa estadístico SPSS-17.0. Se realizaron pruebas de $\chi^{2}$ y de asociación estadística (OR) entre la variable dependiente (cultivo positivo para Malassezia $s p$ ) y las variables independientes (tipo de oreja, edad, género y respuesta inflamatoria por análisis citológico), el valor de significancia establecido fue $(p<0.05)$.

\section{RESULTADOS}

De los 166 caninos que consultaron las diferentes clínicas veterinarias, se evidenció que los pacientes con oreja pendulosa tuvieron una mayor predisposición a la presentación de patologías óticas con un $74.7 \%(n=124)$, estas razas fueron: French Poodle con 19.9\% $(n=33)$, Labrador Retriever con un $16.9 \%(n=28)$ y Golden Retriever con $12.7 \%(n=21)$ en comparación con la razas de oreja erecta; sin embargo, el análisis estadístico no evidenció diferencias significativas entre el tipo de oreja y la presentación de otitis $(p=0.23)$. Con respecto a la edad la mayor predisposición se observó en animales entre 3 y 6 años (37 a 72 meses) donde el porcentaje de patología ótica fue de $25.9 \%$ $(n=43)$, estadísticamente no se observaron diferencias significativas entre los rangos de edades $(p=0.31)$ (Figura 1$)$.

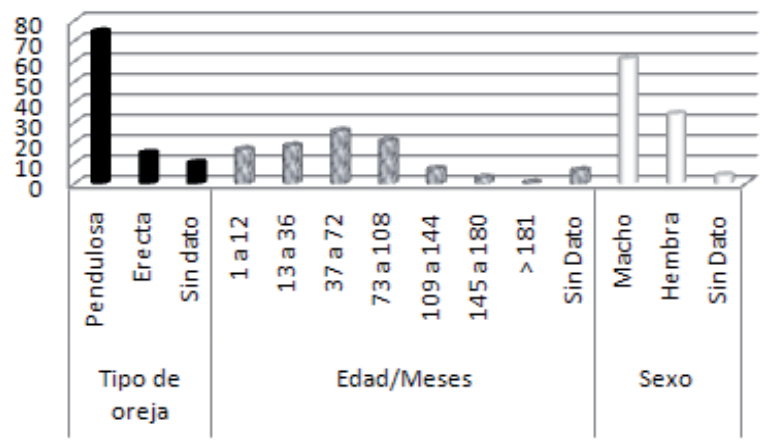

Figura 1. Porcentajes de presentación de otitis con respecto a las variables: Tipo de oreja, edad/meses y sexo.
Se encontró una mayor presentación de otitis en machos $(61.4 \%)$ comparado con las hembras (34.3\%); sin embargo el análisis estadístico no mostró diferencias significativas $(p=0.87)$.

Clínicamente los principales signos evidenciados en los pacientes con oreja pendulosa $(\mathrm{N}=124)$ fueron el prurito con un $80.7 \%(n=100)$, la inflamación en el $73.4 \%(n=91)$ y el exudado oscuro con un $71 \%(n=88)$ como se puede observar en la figura 2.

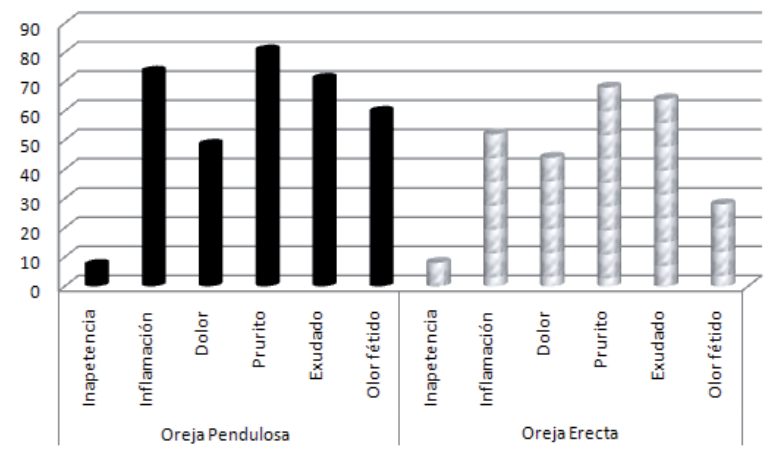

Figura 2. Porcentaje de sintomatología ótica según el tipo de oreja.

A todos los pacientes $(\mathrm{N}=166)$ que ingresaron en este estudio se les realizó análisis citológico a partir de hisopados óticos observándose diferentes grados de reacción inflamatoria mediada principalmente por polimorfonucleares (Tabla 1A). Con respecto a la variable dependiente no se observaron diferencias estadísticamente significativas $(p=0.12)$. En cuanto a la presencia de microorganismos evaluada mediante coloración de Gram se encontraron Bacilos Gram positivos $(n=11)$ y Gram negativos $(n=9)$, así como cocos Gram positivos $(n=64)$ y estructuras con morfología compatible con levaduras $(n=99)$ (Tabla $1 B, C$ y D), con lo que se evidencia que los microorganismos predominantes en el canal auditivo fueron cocos gram positivos y levaduras en una proporción entre ocasionales a $2+$.

Se obtuvo un $59 \%(n=98)$ de muestras positivas para cultivo bacteriológico y el $73 \%(n=121)$ de muestras positivas al cultivo de levaduras (Tabla 1.E); del total de muestras el 28.3\% $(n=47)$ tuvieron aislamientos mixtos. 
Tabla 1. Evaluación citológica y microbiológica de hisopados óticos.

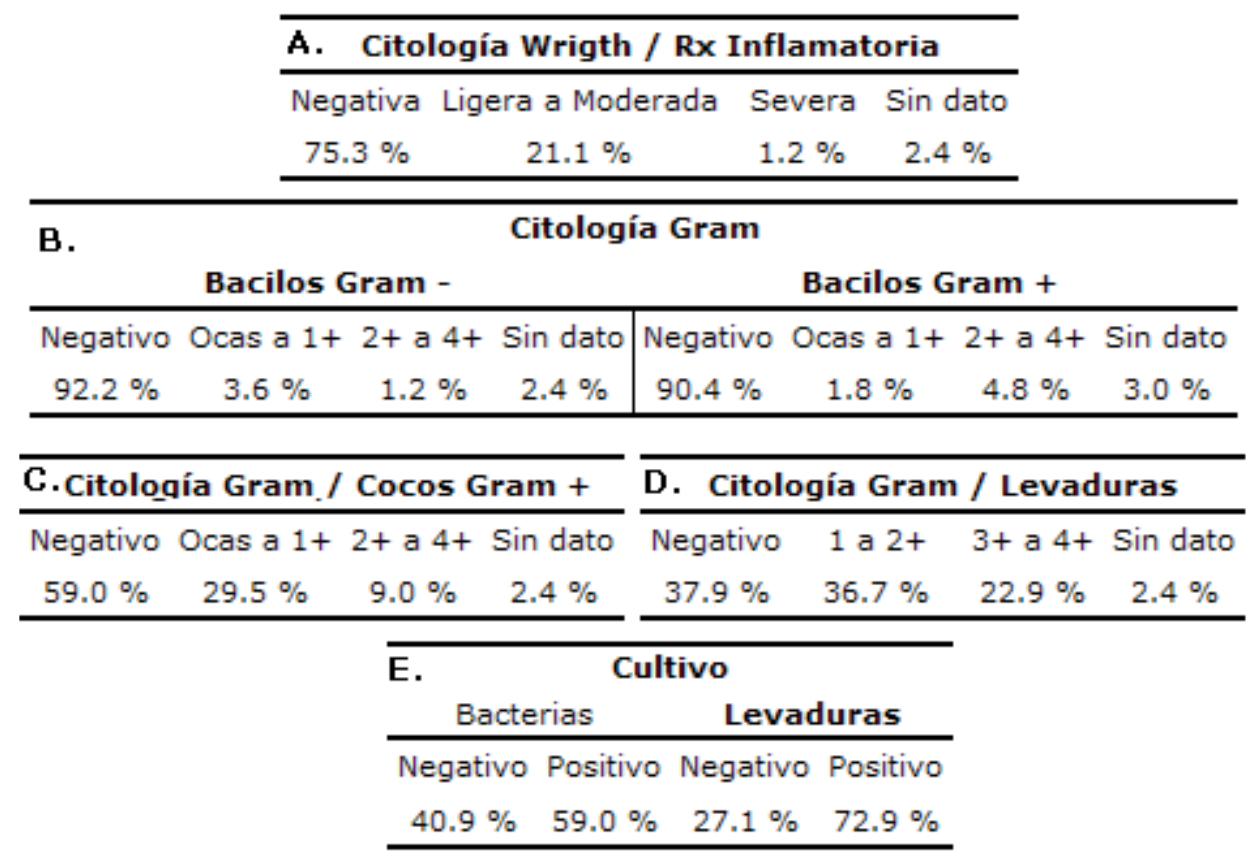

Los aislamientos bacterianos hallados en mayor proporción fueron: Staphylococcus $s p$ en monocultivo con un $36.8 \%$ o en compañía de otros como Streptococcus $\alpha$, $\beta, \gamma$ hemolíticos, Pseudomonas sp, E. coli, Klebsiella $s p$ en el $10.8 \%$, de la misma manera cada uno de estos microorganismos se encontraron en monocultivo o en aislamientos mixtos.
De las 121 muestras con positividad al cultivo (monocultivo o mixto) de levaduras, el $12.4 \%(n=15)$ correspondió Malassezia furfur y a Malassezia pachydermatis el $32.2 \%$ $(n=39)$; los demás aislamientos $52.1 \%$ $(n=63)$ se identificaron como Malassezia spp teniendo en cuenta los patrones descritos por Güehó et al (10) y Guillot et al (11) dadas las reacciones atípicas obtenidas en las pruebas metabólicas realizadas (Figura 3).
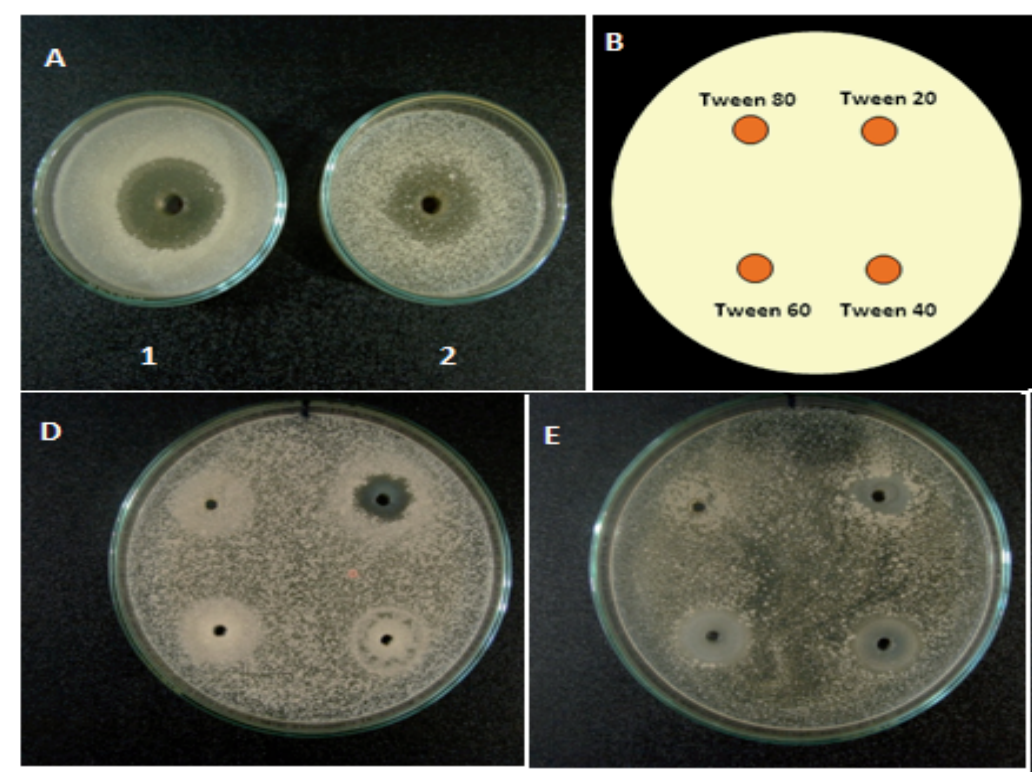
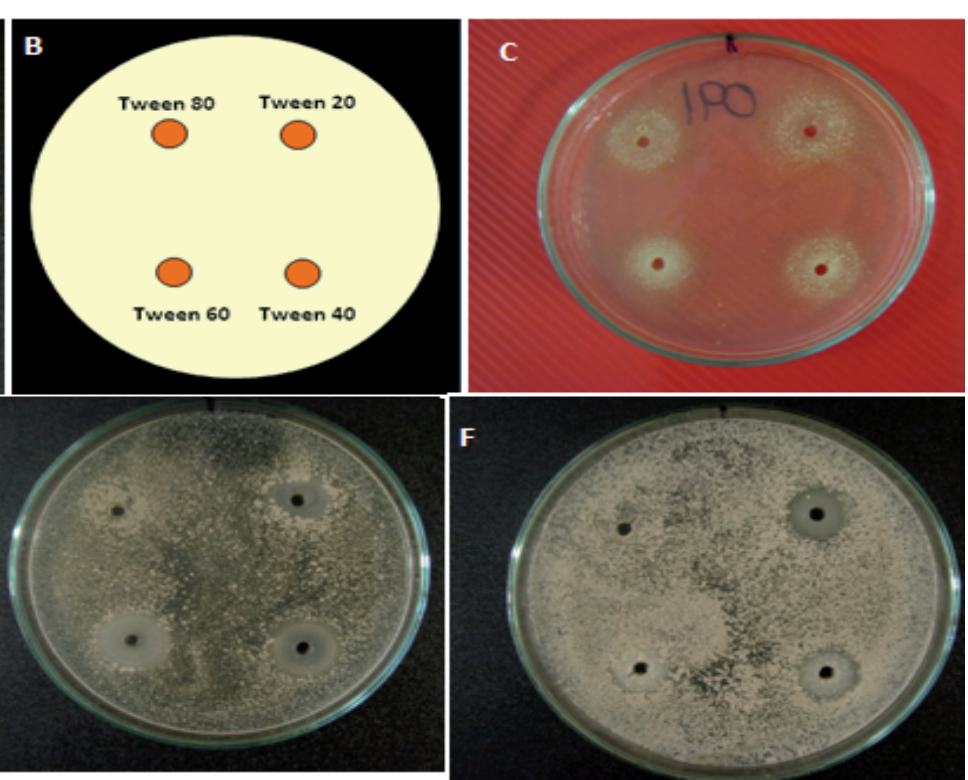

Figura 3. A1. Cremophor negativo, A2. Cremophor positivo. B. Ubicación de los Tweenes en los diferentes pozos. C. Asimilación de todos los Tween. D.Tween 20 negativo. E. Tween 20, 40 y 60 negativos. F. Tween 20 y 40 negativos. 


\section{DISCUSIÓN}

Los hallazgos de acuerdo con el tipo de oreja mostraron una mayor prevalencia de otitis para aquellas razas con orejas pendulosas (74\%) dentro de las cuales se destacan: French Poodle, Labrador Retriever y Golden Retriever con $19.9 \%, 16.7 \%$ y $12.7 \%$ respectivamente; contrario a lo reportado por Crespo et al (13), quienes hallaron un mayor porcentaje de presentación en una raza con oreja erecta como lo es el pastor alemán (20.2\%), seguida por razas criollas y Cocker Spaniel con $19.7 \%$ y $15.9 \%$ respectivamente, al igual que los reportado por Nobre et al (17) quienes encontraron un $92.3 \%$ de los pacientes con orejas erectas con cultivos positivos a Malassezia pachydermatis, mientras los de oreja pendulosa correspondieron al $71.6 \%$.

De acuerdo con los resultados obtenidos en este estudio, no se encontraron diferencias estadísticamente significativas entre la presentación de otitis por Malassezia $s p$ relacionada con el tipo de oreja del paciente; estos resultados concuerdan con lo reportado por Masuda et al (6), quienes tampoco reportan esta diferencia.

El rango de edad donde se halló una mayor proporción de problemas óticos se dio entre los 3-6 años $(25.9 \%)$, lo que concuerda parcialmente con lo observado por Girao et al (7) quienes obtuvieron rangos de mayor presentación en edades de 1 - 3 años $(44.7 \%)$ y de 4 - 6 años $(35.7 \%)$; en el análisis estadístico no se observaron diferencias significativas en cuanto a la variable edad al igual que lo observado por Crespo et al (5).

En cuanto a la variable género al igual que en diferentes reportes no se observaron diferencias significativas $(5,7)$.

Con respecto a la sintomatología clínica, los resultados arrojaron en su orden un mayor porcentaje de presentación para prurito $(80.7 \%)$, inflamación $(73.4 \%)$ y exudado $(71.0 \%)$ independientemente del tipo de oreja; mientras que Masuda et al (6), consideran como principal signo la abundante secreción ótica con un $79.16 \%$; por su parte Nobre et al (17), reportaron que los signos predominantes fueron eritema y secreción con un $51.3 \%$. En general se pudo observar una concordancia en cuanto a los principales signos clínicos de pacientes con otitis en este estudio, al compararlo con otras investigaciones internacionales.

Malassezia pachydermatis, ha sido frecuentemente implicada como el principal agente etiológico de las otitis externas en caninos, así lo afirman Crespo et al (5), Nobre et al (17), Sallerller y Kirkan (18), Petrov y Mihaylon (19), Oliveira et al (20) al reportarla en casos clínicos en un $62.2 \%, 76.5 \%$ y $73 \%$ respectivamente; los resultados obtenidos en este análisis comparten parcialmente lo observado por estos autores, aunque el porcentaje de asilamiento de $M$. pachydermatis fue de $32.3 \%$; sin embargo, esto contrasta con otros reportes como el de Sarierler y Kirkan (18), quienes hallaron porcentajes muy bajos de esta especie (5.1\%). Por otra parte, el $52.1 \%$ correspondió a Malassezia $s p$, dado que los patrones de asimilación metabólica no coincidieron con los perfiles establecidos por Guého et al (10); Guillot et al (11) y Mayser et al (16). Con respecto a las especies lipodependientes se halló un $12.4 \%$ de aislamientos correspondientes a Malassezia furfur frente al $4.5 \%$ reportado en otras investigaciones $(5,7,19)$.

A diferencia de lo obtenido por otros autores como Nobre et al (17), Oliveira et al (20), Sarierler y Kirkan (18) donde Candida albicans fue encontrada en un $2 \%, 3 \%$ y $12.82 \%$ respectivamente, en el presente estudio no se presentó ningún aislamiento.

Los aislamientos de tipo bacteriano implicados en los procesos óticos correspondieron principalmente a Staphylococcus $s p$ con un $36.8 \%$, siendo esta bacteria reconocida como parte de flora normal; la asociación con Malassezia pachydermatis se dio en un $19.9 \%$ de los casos analizados, mientras para otros autores esta asociación se dio en porcentajes altos como del $54.8 \%$ (20), pero coinciden con los reportado por Nobre et al (17), donde la relación está en un $19.2 \%$ y con Petrov y Mihaylov (19), quienes obtuvieron un $18.8 \%$. 
En aislamientos adicionales, el $10.8 \%$ fue identificado como Streptococcus $\alpha, \beta$, $\gamma$ hemolíticos, Pseudomonas sp, E. coli, Klebsiella $s p$ y Proteus $s p$ hallados en cultivo puro o en asociaciones entre ellos y/o con Staphylococcus $s p$, hallazgos acordes con el $12 \%$ reportado por Oliveira et al $(20)$; sin embargo, contrasta con el $37.5 \%$ de Petrov y Mihaylov (19) y el $33.4 \%$ de Nobre et al (17), aunque coinciden los géneros bacterianos reportados, dentro de los cuales Pseudomonas $s p$ es reconocido como el de mayor implicación dada sus características patogénicas. Todos estos agentes pueden llegar a complicar la presentación de la enfermedad llevando a otitis recurrentes como se evidenció en estudios preliminares realizados por el grupo de investigación, en los que el porcentaje de recurrencia observado fue $12.5 \%$.

En conclusión, se evidenció que la presentación de la patología es independiente del género, la edad y la raza; así mismo la severidad de la otitis es independiente del tipo de oreja; con respecto a las manifestaciones clínicas que predominaron fueron el prurito, la inflamación y el exudado oscuro quedando descartada como signo típico la inapetencia.
Se estableció la implicación de las levaduras pertenecientes al género Malassezia $s p$ en los casos de otitis externa en caninos de Bogotá con un $73 \%$ en ocasiones asociadas a agentes bacterianos causando sintomatología más severa.

Siendo este el primer estudio realizado en Colombia sobre el aislamiento de Malassezia $s p$ en caninos con problemas óticos, se demuestra la importancia del diagnóstico microbiológico con el fin de establecer el/los agentes etiológicos involucrados; adicionalmente si se tienen en cuenta los resultados obtenidos es necesario realizar una identificación molecular para determinar las especies de este género de levaduras implicadas en la epidemiología de las otitis en el país.

\section{Agradecimientos}

Dr. Carlos Moreno T. y Dr. Olimpo Oliver E., Universidad Nacional de Colombia, Laboratorio Clínico de la Facultad de Medicina Veterinaria y de Zootecnia, a la Clínica de pequeños animales, ABANIMAL, al Centro de Especialidades Veterinarias (CEV) y Zoovida (EPSA) y al Laboratorio de Micología, Pontificia Universidad Javeriana.

\section{REFERENCIAS}

1. Matousek JL. Ear disease. Vet Clin North Am Small Anim Pract 2004; 34:xi-xii.

2. Rosser EJ. Causes of otitis externa. Vet Clin North Am Small Anim Pract 2004; 34:459-468.

3. Bernardo FM, Martins HM y Martins ML. A survey of mycotic otitis externa of dogs in Lisbon. Rev Iberoam Micol 1998; 15:163-165

4. Guillot J. y Bond R. Malassezia pachydermatis: A review. Med Mycol 1999; 37:295-306.
5. Crespo MJ, Abarca ML y Cabañes FJ. Occurrence of Malassezia spp in the external ear Canals of dogs and cats with and without otitis externa. Med Mycol 2002; 40:115-121.

6. Masuda A, Sukegawa T, Mizumoto N, Tani $\mathrm{H}$, Miyamoto $T$, Sasai $\mathrm{K}$ and Baba E. Study of lipid in the ear canal in canine otitis externa with Malassezia pachydematis. J Vet Med Sci 2000; 62(11):1177-1182. 
7. Girão $M D$, Prado $M R$, Brihante RSN, Cordeiro RA, Monteiro AJ, Sidrim JJC, Rocha MFG. Malassezia pachydermatis isolated from normal and diseased external ear Canals in dogs: A comparative analysis. Br Vet J 2006; 172: $544-548$.

8. Angus JC. Otic cytology in health and disease. Vet Clin North Am Small Anim Pract 2004; 34: 411-424.

9. Aizawa T, Kano R, Nakamura $Y$, Watanabe S, Hasegawa A. Molecular heterogeneity in clinical isolates of Malassezia pachydermatis from dogs. Vet Microbiol 1999; 70:67-75.

10. Guého E, Midgley G \& Guillot J. The Genus Malassezia with description of four new species. Antonie van Leeuwenhoek 1996; 69:337-355.

11. Guillot J, Guého E, Lesourd M, Midgley G, Chévrier G, Dupont B. Identification of Malassezia species. J Mycol Med 1996; 6:103-110.

12. Giusiano G. Malassezia, Estado del conocimiento y perspectivas en su estudio. Rev Argent Microbiol 2006; 38: $41-48$

13. Crespo M J, Abarca M L \& Cabañes $F$ J. A typical lipid-dependent Malassezia species isolated from dogs with otitis externa. J Clin Microbiol 2000; 38(6):2383-2385.

14. Kaneko T, Makimura K, Abe M, Shiota R, Nakamura $Y$, Kano R, Hasegawa A, Sugita T, Shibuya S, Watanabe S, Yamaguchi $\mathrm{H}$, Abe $\mathrm{S}$ and Okamura N. Revised culture-based system for identification of Malassezia sp. J Clin Microbiol 2007; 14(11):337-342.
15. MacFaddin J F. Pruebas bioquímicas para la identificación de bacterias de importancia clínica. 4a Ed. Buenos Aires, Argentina: Editorial Panamericana; 1980.

16. Mayser $P$, Haze $P$, Papavassilis $C$, Pickel M, Gruender K, Guehó E. Differentitation of Malassezia species: selectivity of cremophor EL, castor oil and ricinoleic acid for M. furfur. Br J Dermatol 1997; 137:208-213.

17. Nobre M O, Castro A P, Nascente P S, Ferreiro $L$, Meireles M C A. Occurrence of Malassezia pachydermatis and other infectious agents as cause of external otitis in dogs from Rio grande do sul state, Brasil (1996/1997). Braz J Microbiol 2001; 32:245-249.

18. Sarierler M and Kirkan S. Microbiological diagnosis and therapy of canine otitis externa. Veteriner Cerrahi Dergisini (JTVS) 2004; 10(3-4):11-15.

19. Petrov V y Mihaylov G. Malassezia pachydermatis - Etiology and clinical findings in canine external otitis therapeutic approaches. Trakia Journal of Sciences (TJS) 2008; 6(Suppl 1):123-126.

20. Oliveira $C L$, Leite $C A L$, Brilhante $R S N$, Carvalho C BM. Comparative study of the microbial profile from bilateral otitis externa. Can Vet J 2008; 49:785-788. 